\title{
DETECTION OF EXOPLANETS IN M31 WITH PIXEL-LENSING: THE EVENT PA-99-N2 CASE
}

\author{
G. INGROSSO* and F. DE PAOLIS \\ Dipartimento di Fisica, Università del Salento and INFN Sezione di Lecce, Italy, \\ *E-mail: ingrosso@le.infn.it \\ S. CALCHI NOVATI \\ Dipartimento di Fisica, Università di Salerno and INFN Sezione di Napoli, Italy \\ PH. JETZER \\ Institute for Theoretical Physics, University of Zürich, Switzerland \\ A. A. NUCITA \\ XMM-Newton Science Operations Centre, ESAC, ESA, Madrid, Spain \\ A. F. ZAKHAROV \\ ITEP, Moscow, and BLTP, JINR, Dubna, Russia
}

\begin{abstract}
We show that exoplanets in the M31 galaxy may be detected with the pixel-lensing method by using telescopes making high cadence observations of an ongoing microlensing event. Although the mean mass for detectable exoplanets is about $2 M_{\mathrm{J}}$, even small mass exoplanets $\left(M_{\mathrm{P}}<20 M_{\oplus}\right)$ can cause significant deviations, which are observable with large telescopes. We reanalysed the POINT-AGAPE microlensing event PA-99-N2. First, we test the robustness of the binary lens conclusion for this light curve. Second, we show that for such long duration and bright microlensing events, the efficiency for finding planetary-like deviations is strongly enhanced.
\end{abstract}

Keywords: Gravitational Lensing, Galaxy: halo, Galaxies: individuals: M31

The possibility to detect planets in pixel-lensing observations towards M31 has been already explored earlier. The analysis for planet detection, however, has been performed by using a fixed configuration of the underlying Paczyński light curve. Using a Monte Carlo (MC) approach we study the chances to detect exoplanets in M31, by considering the multi-dimensional space of parameters for both lensing and planetary systems ${ }^{1}$ The method of residuals allows us to select the light curves that show detectable deviations with respect to the Paczyński shape (describing microlensing by single lens). The advantage of the MC approach is that of allowing a detailed characterisation of the sample of microlensing events for which the planetary deviations are more likely to be detected. We discriminate two classes 
of events (indicated by I and II), according to the ratio $\rho / u_{0}>1$ or $\rho / u_{0}<1$. The events of the I class have short durations $\left(\left\langle t_{1 / 2}\right\rangle \simeq 1.6\right.$ day) and larger flux variations $\left(\left\langle R_{\max }\right\rangle \simeq 20.6 \mathrm{mag}\right)$. In these events, planetary deviations are caused by the source trajectory crossing (in the lens plane) the central caustic region, close to the primary lens star. The events of the II class have longer durations $\left(\left\langle t_{1 / 2}\right\rangle=6.4\right.$ day) and smaller flux variations $\left(\left\langle R_{\max }\right\rangle=23.1 \mathrm{mag}\right)$. In this case planetary perturbations are (mainly) caused by the intersection of the source trajectory with the planetary caustic regions and may also appear at times far from the maximum amplification time $t_{0}$. The fraction of I class events is about $5 \%$ of all generated events. However, it turns out that the probability to have detectable planetary features in these events (that however are rare) is higher. This happens since the crossing of the central caustic region is more probable in I class events (with $\rho / u_{0}>>1$ ). On the contrary, the generated events of the II class are more numerous, but have a smaller probability to show detectable planetary features. An example of II class event is given in Fig. 1, where we see that also a small mass planet $\left(M_{\mathrm{P}}=0.3 M_{\oplus}\right)$ can cause detectable planetary deviations in events for which the finite size effects are small. In the simulated event the geometry is such that the source trajectory is passing (in the lens plane) in the region between the two planetary caustics, where a deficit of amplification is present. Other examples of light curves for I and II class events are given in paper. ${ }^{1]}$

Our analysis shows (see Fig. 2, upper panel) that, in spite of the initial distributions, larger planetary masses lead to higher probability for the detection of planetary features in light curves. We also find that the planet detection is maximized when the planet-to-star separation $d_{\mathrm{P}}$ is inside the "lensing zone" (see Fig. 2 , bottom panel). Typical duration of planetary perturbations is about 1.4 days. However, the number of significant planetary deviations on each light curve increases with increasing ratios $\rho / u_{0}$. So, the overall time scale for planetary deviations can increase up to $3-4$ days (for I class events). This means that a reasonable time step for pixel-lensing observations aiming to detect planets in M31 is a few (4-6) hours. Among the 25 pixel-lensing events discovered up to now, ${ }^{2}$ the POINT-AGAPE collaboration ${ }^{3}$ reported the detection of the event PA-99-N2. This appears as a peculiar event because of its extreme brightness $\left(R_{\max } \simeq 19 \mathrm{mag}\right)$, long duration $\left(t_{1 / 2} \simeq 24\right.$ day) and location, some 22 arcmin away from the M31 center. Furthermore, as it has been shown in a subsequent analysis $\stackrel{4}{,}$ the light curve can be attributed to a secondary component orbiting the lens star, because of the anomaly with respect to the Paczyǹki shape. In particular, An et al. ${ }^{[4}$ have evaluated the a posteriori probability distribution for the lens mass which results to be extremely broad: for source and lens disc objects, they report the lens mass range $0.02-3.6 M_{\odot}$ at $95 \%$ confidence level. Together with the small binary lens mass ratio, $q \simeq 1.2 \times 10^{-2}$ for the best fit, this puts the lens companion well in the substellar range. Ingrosso et al ${ }^{1}$ have remarked that taking the likely value for the lens mass $\simeq 0.5 M_{\odot}$ for a disc lens, the lens companion would be $\mathrm{a} \simeq 6.34 M_{\mathrm{J}}$ object. This would make of the PA-99-N2 lens companion the first exoplanet discovered in M31. Furthermore, we 

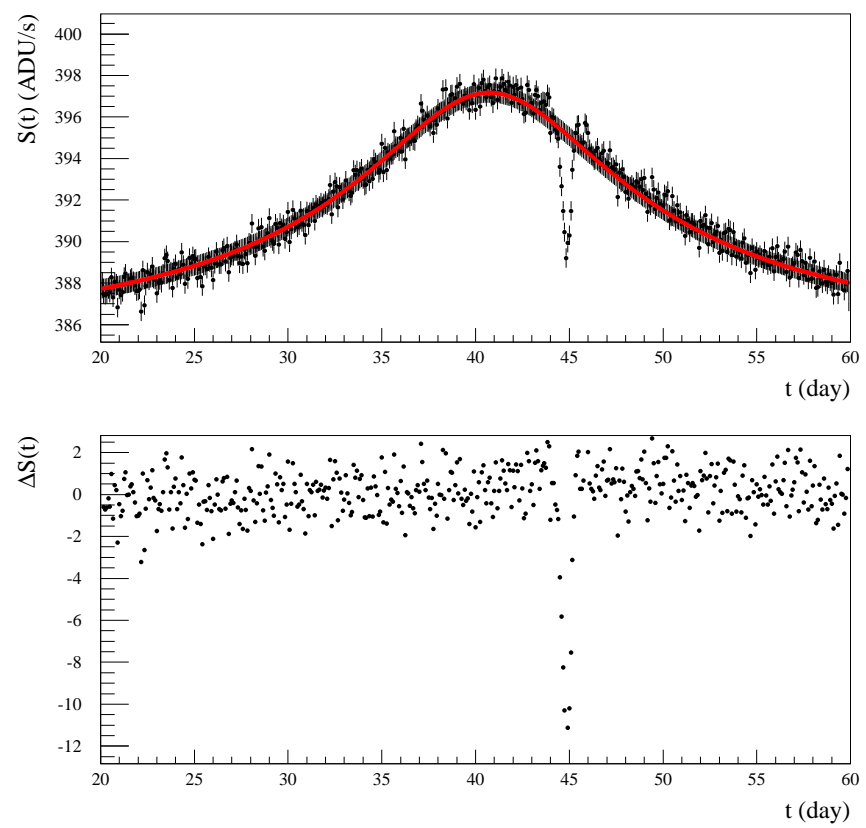

Fig. 1. Simulated II class event (points) and Paczyǹki fit (thin solid line). Some parameter values: $\rho / u_{0}=0.04, R_{\max }=24.0 \mathrm{mag}, t_{1 / 2}=18.7$ day, $M_{\mathrm{P}}=0.3 M_{\oplus}, d_{\mathrm{P}}=3.8 \mathrm{AU}$.

had analysed the PA-99-N2 event within the framework of our simulation scheme, showing in particular that its parameters nicely fall in the expected range for II class events $!^{[1}$ We further analyse this event.$^{[5}$ First, starting from the observational data (courtesy of the POINT-AGAPE collaboration), we test the robustness of the binary-lens best fit solution. Second, we address the question of the efficiency for finding binary-like deviations for such bright and long duration events. To verify the robustness of the binary-lens fit solution, the first test is to add a Gaussian noise to the best fit of the observed light curve to verify if a single lens model with noise can reproduce the observational data. Similarly, we have taken the best fit binary model (named $\mathrm{C} 1$ in Table 1 of An et al ${ }^{4}$ ) and realised more than one thousand models by adding Gaussian noise and letting the parameters to vary by at most $20 \%$. For each of the so obtained light curves (107 data points), we have verified that the $\chi^{2}$ values for single lens models ( 8 parameters) are greater (by a factor of about 3 ) with respect to those corresponding to binary fit models (11 parameters). We also find that the two $\chi^{2}$ distributions are clearly separated, which implies that the best single lens fit is much worst than any of the binary lens models. From this we conclude that the binary fit is robust and that the observed light curve cannot be fitted by any single lens model with random noise. 

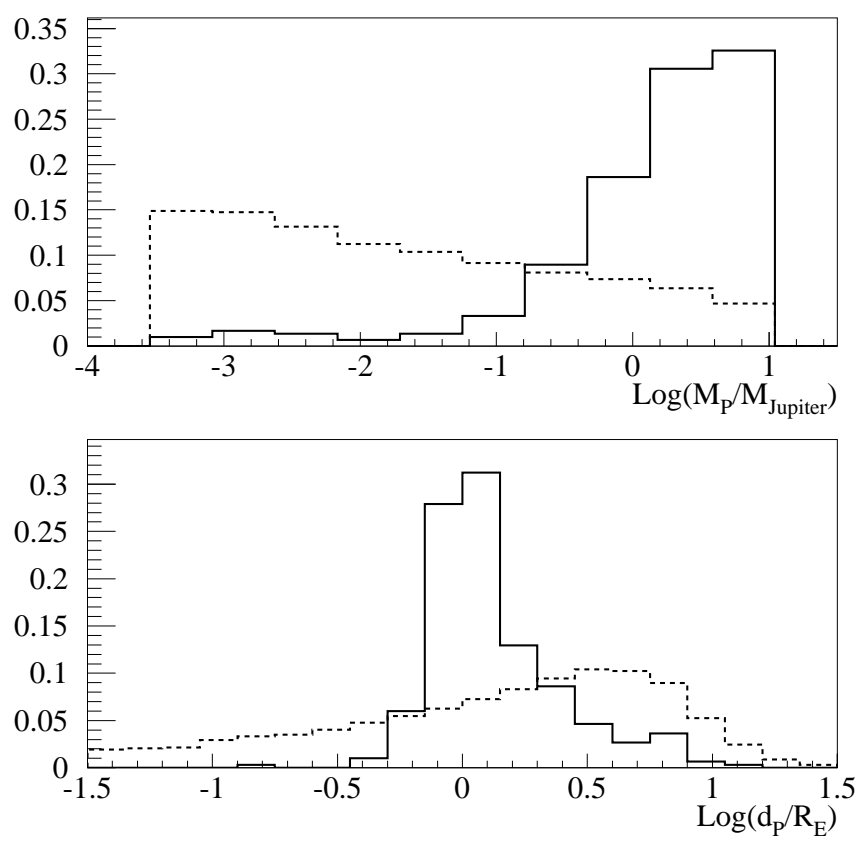

Fig. 2. Upper panel: distribution of the planet mass $M_{\mathrm{P}}$ for the events with detectable planetary deviations (solid line) and for the generated events (dashed line). Bottom panel: distribution of $d_{\mathrm{P}} / R_{\mathrm{E}}$ for events as before.

A further point to be stressed is the following: we mentioned that the probability to detect an exoplanet in pixel-lensing observations towards M31 is rather small, even with large telescopes ${ }^{11}$ Therefore, the question which arises now is the following: what is the chance of finding a planetary feature in an event as PA-99-N2? The basic answer can be found looking at the characteristics of the underlying microlensing event: this is, at the same time, an extremely bright and long duration event. In fact, as we now show, this strongly enhances the probability for finding deviations to the single lens shape. To this purpose we perform a MC simulation where we fix the single lens parameters $R_{\max }$ and $t_{1 / 2}$ to those of PA-99-N2 and we let vary the binary ones (planet-to-star mass ratio, planetary distance and orientation of the source trajectory with respect to the binary axis) for an observational setup fixed to reproduce these POINT-AGAPE observations. For the selected planetary mass range we find an increase of the average efficiency up to $6 \%$, to be compared with $0.6 \%$ (see Table 3 in paper ${ }^{1}$ ) for events without any constraints on $R_{\max }$ and $t_{1 / 2}$. The efficiency for finding binary-like deviations in the simulated light curves increases significantly with the lens-companion mass value. In particular, for the restricted range $1-10 M_{\mathrm{J}}$ the average efficiency rises up to $27 \%$ of the generated 


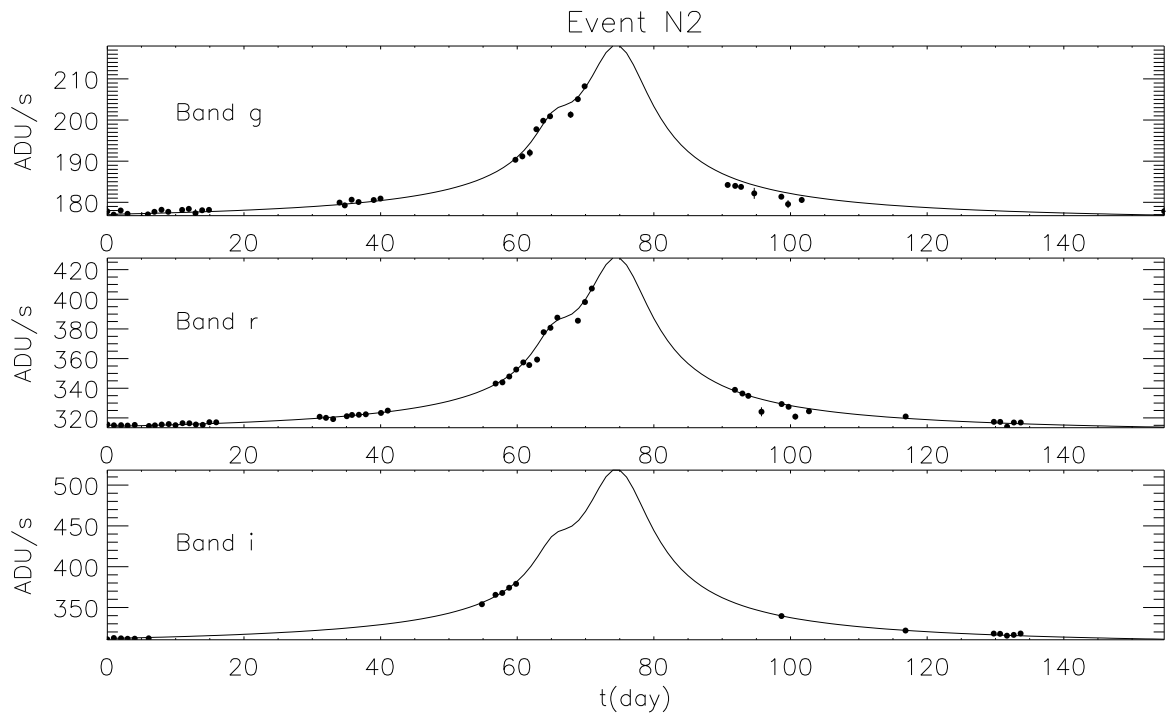

Fig. 3. The binary light curves corresponding to the $\mathrm{C} 1$ mode $\sqrt{4}$ for $\mathrm{g}, \mathrm{r}$ and i bands.

events. We have restricted our attention to the planetary-mass range for the lens companion, but of course we expect the efficiency still rises when we include the brown-dwarf mass range (as in fact it was allowed by the analysis ${ }^{4}$ ).

This further analysis shows that the probability to find exoplanets in M31, at least in the Jupiter mass range, with the pixel-lensing technique is not so small as previously thought and thus that it is now mature for implementation. Clearly, the use of telescopes with wide fields of view is needed in order to get a large enough number of events and thus to get sufficient statistics. Actually, a detection of planetary features in pixel-lensing events may be done also with telescopes of small field of view, if early warning systems (similarly to the OGLE one) will be in operation. Finally, we remark that gravitational microlensing is a very efficient method for discovering exoplanets around habitable zone, namely planetary systems with Earth-like masses and distances of about AU.

\section{References}

1. Ingrosso, G. et al., MNRAS, 399, 219 (2009)

2. Calchi Novati, S., GRG, in press

3. Paulin-Henriksson, S. et al., A\&A, 405, 15 (2003)

4. An J.H., et al., ApJ, 601, 845 (2004)

5. Ingrosso, G. et al., GRG, in press 